\title{
SOME FEATURES IN THE ANATOMY AND LATER DEVELOPMENT OF THE HEAD OF DELPHINUS DELPHINUS LINNÉ.
}

\author{
By H. Leighton Kesteven, D.Sc., M.D., Ch.M., \\ Honorary Zoologist, The Australian Museum.
}

(Figures 1-29.)

Part I.-OSTEOLOGY.

This part of the work is based on two foetal heads which I received from the New South Wales Fisheries Commission some years ago.

The larger of these was converted into a skull by careful dissection, and it has now been extensively disarticulated as the work proceeded. It measured seventy-eight millimetres from tip to occiput.

The smaller was decalcified and cut into serial sections along the sagittal plane after straining in alum carmine. This specimen measured fifty-two millimetres from
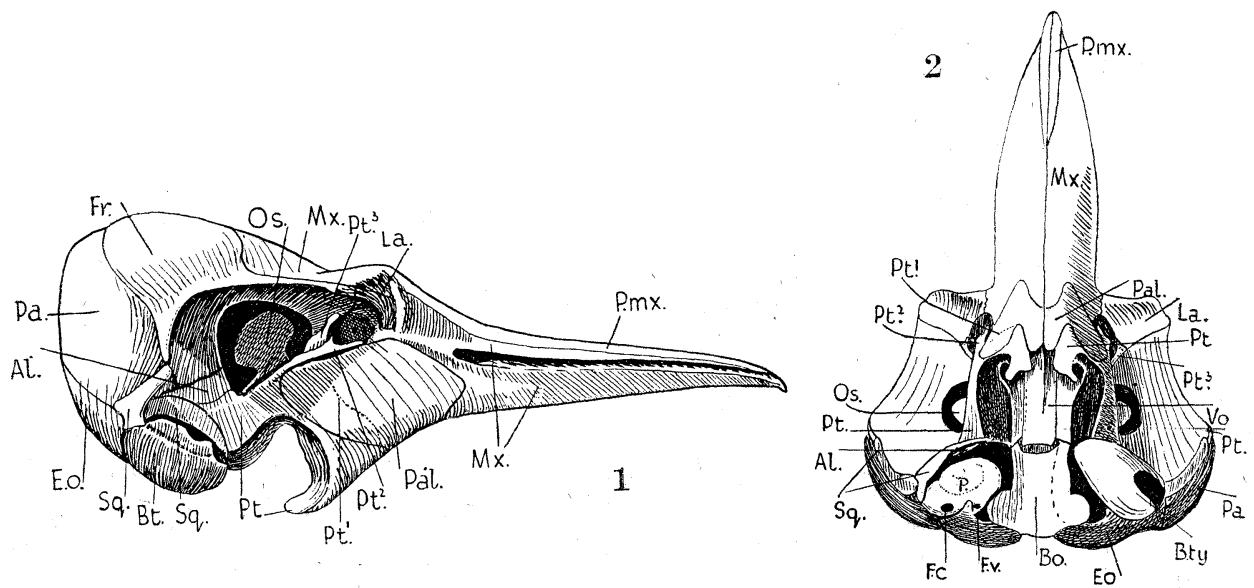

Fig. 1.-Delphinus delphinus Linné. The skull of a $78 \mathrm{~mm}$. embryo, lateral view.

Fig. 2.-The same, ventral view.

\section{Abbreviations used on illustrations to Part I.}

$\mathrm{A}^{1}$ \& $\mathrm{A}^{2}$, Accessory tympanic ossicle. Al., Alisphenoid. A.o., Ala orbitalis. Aq.coch., Aqueductus cochleae. Bo., Basioccipital. B.s., Basisphenoid. Bs.o., Basisphenoid ossific centre. Bt. \& Bty., Basitympanic. C.n.l.d., Cartilage of the naso-lachrymal duct. E.o., Exoccipital. Fen.c., Fenestra cochleae. F.o., Fenestra ovale. Fr., Frontal. F.v., Fenestra vestibuli. I., Inous. La., Lachrymal. Le., Lateral ethmoidal plate of the vomer. L.t.a. \& L.t.p., Lamina transversalis anterior and posterior. M., Malleus. Me., Mesethmoid. Mx., Maxilla. Na., Nasal. Os., Orbitosphenoid. Pa., Parietal. P., Pet. \& Petr., Petrosal. Pl.e., Planum ethmoidale. P.mx., Premaxilla. Po., Postorbital process of the frontal. Ps., Presphenoid. Ps.a. \& Ps.p., Anterior and posterior paraseptal cartilages. Ps.o., Presphenoidal ossific centre. Pt., Pterygoid.. Pt., Pterygoid and Palatine suture. Pt., Pterygoid, processus anterior. Pt., Pterygoid, processus anterior, lower limb. Pt., Pterygoid, processus anterior, upper limb. R., Rostrum. Sn., Septum nasi. Sq., Squamosal. St., Stapes, T.n., Tectum nasi. Ty., Tympanic bone. Vas., Vascular foramina. Vo., Vomer. VII, Facial canal. 